Canad. Math. Bull. Vol. 19 (1), 1976

\title{
ON THE PROPERTY (PU) FOR *-REGULAR RANK RINGS
}

\author{
BY \\ JOHN L. BURKE
}

Introduction. In this paper we consider an irreducible *-regular ring $\mathscr{R}$ with order $k$ for some $k \geq 4$. If $\mathscr{R}$ is also a Baer ring it is a rank ring. Our first result is:

THEOREM 1.3. Let $\mathscr{R}$ be an irreducible *-regular Baer ring with order $k$ for some $k \geq 4$. The following are equivalent.

(i) For any e, $f \in P(\mathscr{R}), \stackrel{a}{\sim} f \Rightarrow e \stackrel{*}{\sim} f$.

(ii) For any $e, f \in P(\mathscr{R}), \stackrel{a}{\sim} f \Rightarrow e \stackrel{u}{\sim} f$.

(iii) For any $a \in \mathscr{R}, P_{a} \stackrel{*}{\sim}_{a} P$.

(iv) For any $e, f \in P(\mathscr{R}), e \cup f-f \stackrel{*}{\sim}_{e}-e \cap f$.

(v) If $e \in P(\mathscr{R})$ and $x^{*} x \in e \mathscr{R} e$, then there exists $z \in e \mathscr{R} e$ with $x^{*} x=z^{*} z$.

We give the name "property $(P U)$ " to (i) of Theorem 1.3, and express our second result as:

TheOREM 3.1. Let $\mathscr{R}$ be an irreducible *-regular Baer ring with order $k, k \geq 4$, which satisfies property $(P U)$. Then property $(P U)$ lifts from $\mathscr{R}$ to $\mathscr{R}_{n}$.

The ring $\mathscr{R}$ can be a rank ring without being a Baer ring. In this case, the completion $\mathscr{R}^{\wedge}$ of $\mathscr{R}$ in rank metric is a Baer ring. Our third result is:

THEOREM 6.1. Let $\mathscr{R}$ be an irreducible $*$-regular rank ring with order $k, k \geq 4$, in which comparability holds and which satisfies property $(P U)$. Then property $(P U)$ extends from $\mathscr{R}$ to $\mathscr{R}^{\wedge}$.

We conclude with an application to rank metric completions of certain inductive limits.

1. Preliminaries. A ring $\mathscr{R}$ is regular if the equation axa=a is soluble in $\mathscr{R}$ for any $a$ in $\mathscr{R}$. A $*$-regular ring is a regular ring $\mathscr{R}$ which admits an involution with the property that for any $a \in \mathscr{R}, a^{*} a=0$ implies $a=0$. We say that an irreducible regular ring is discrete if its projection lattice is atomic; otherwise, it is continuous. The rings with which we will be concerned will have a unit and will either be continuous rings or discrete rings with order $k$ for some $k \geq 4$ [7, Definition 3.6,p.100]. A ring is a Baer ring if the left and right annihilators of every subset are generated by idempotents. A Baer ring has a unit and a *-regular ring is a

Received by the editors January 14, 1972 and, in revised form, December 13, 1972. 
Baer ring if and only if it is complete (that is, its lattice of projections is a complete lattice). If a ring is regular but not Baer it may fail to have a unit element; in this case, we will always specify the existence of a unit element. A rank ring is a regular ring $\mathscr{R}$ which admits a rank function. That is, there exists a real-valued function $R(a), a \in \mathscr{R}$, such that:

(i) $0<R(a)<\infty$ for all $a \neq 0$;

(ii) $R(a b) \leq R(a), R(b)$;

(iii) If $e$ and $f$ are orthogonal idempotents then $R(e+f)=R(e)+R(f)$.

If $\mathscr{R}$ has a unit element and $R(1)=1$ the rank function is said to be normalized. An irreducible $*$-regular Baer ring admits a normalized rank function. In fact:

THEOREM 1.1. An irreducible *-regular ring $\mathscr{R}$ with order $k, k \geq 4$, is a Baer ring if and only if it admits a normalized rank function with range contained in the closed unit interval and is complete in rank metric.

Proof. Suppose that $\mathscr{R}$ is Baer. Then the lattice of projections (which we identify with the lattice of principal right ideals) of $\mathscr{R}$ is a continuous geometry (see [4]). A continuous geometry admits a dimension function $D$ with range contained in the closed unit interval [7, Theorem 6.9, p. 52]. For $a \in \mathscr{R}$ define $R(a)$ by $R(a)=D(e)$ where $e$ is the unique projection which generates the principal right ideal of $a$. This function $R: \mathscr{R} \rightarrow[0,1]$ is a rank function and $\mathscr{R}$ is complete in rank metric [7, Theorem 17.4 , p. 230].

Suppose that $\mathscr{R}$ admits a rank function with range contained in the closed unit interval and is complete in rank metric. Then $\mathscr{R}$ has a unit [1, Theorem 3.7 (iv), p. 716]. Thus, [7, Theorem 18.1, p. 237] applies to yield the result claimed.

This completes the proof.

We call an element $x$ of a $*$-regular ring $\mathscr{R}$ positive if $x$ has the form $a^{*} a$ for some $a \in \mathscr{R}$. We denote the lattice of projections of $\mathscr{R}$ by $P(\mathscr{R})$. If $e, f \in P(\mathscr{R})$ and there exists $x \in e \mathscr{R} f$ and $y \in f \mathscr{R} e$ with $e=x y$ and $f=y x, e$ and $f$ are algebraically equivalent; in case $e$ and $f$ are algebraically equivalent, we write $\stackrel{a}{\sim} f$. If there exists $w \in e \mathscr{R} f$ (to be called partial unitary) with $e=w w^{*}$ and $f=w^{*} w, e$ and $f$ are $*$-equivalent; in this case we write $e^{*} \sim f$. If $e$ and $f$ are exchangeable by a unitary, that is, if there exists $u \in \mathscr{R}$ with $u u^{*}=u^{*} u=1$ and $u e u^{*}=f$, we say that $e$ and $f$ are unitarily equivalent; in this case we write $e \stackrel{u}{\sim} f$. If $e$ and $f$ have a common complement in $P(\mathscr{R})$ they are perspective; in this case we write simply $e \sim f$.

We denote by $a \mathscr{R}$ the right ideal generated by the element $a$ of a $*$-regular ring $\mathscr{R}$ and we denote by $p_{a}$ the unique projection satisfying $a \mathscr{R}=p_{a} \mathscr{R}$; we call $p_{a}$ the left projection of $a$. Left ideal is denoted and right projection is defined analogously; we denote by ${ }_{a} p$ the right projection of $a \in \mathscr{R}$. If $e, f \in P(\mathscr{R})$ we denote by $e \cup f$ and $e \cap f$ their lattice join and their lattice meet, respectively, in $P(\mathscr{R})$. If $e, f \in$ $P(\mathscr{R})$ are orthogonal, $e \cup f=e+f$; if $e, f \in P(\mathscr{R})$ and $e \leq f$, then $e=f-g$ where $g \in P(\mathscr{R})$ is the orthogonal complement of $e$ in $f$. 
Definition. We say that a $*$-regular ring $\mathscr{R}$ has property $(P U)$ if any two algebraically equivalent projections in $\mathscr{R}$ are $*$-equivalent.

It is well-known that not every *-regular ring has property $(P U)$; we give an example below of a *-regular ring which does not.

ExAmple. Let $Q$ denote the field of rational numbers and $Q_{4}$ the ring of $4 \times 4$ matrices over $Q$. This ring is an irreducible *-regular Baer ring which has order 4 . Moreover, the ring has infinitely many elements and the centre (namely, $Q$ ) has characteristic zero. It does not have property $(P U)$. Let the matrices $E=\left(e_{i j}\right)$, $F=\left(f_{i j}\right), X=\left(x_{i j}\right), Y=\left(y_{i j}\right) \in Q_{4}$ be defined by:

$$
\begin{array}{cl}
e_{i j}=\left\{\begin{array}{ll}
\frac{1}{5}, & i=j=1 \\
\frac{2}{5}, & i=1, j=2 \\
\frac{2}{5}, & i=2, j=1 \\
\frac{4}{5}, & i=j=2 \\
0, & \text { otherwise; }
\end{array} \quad f_{i j}= \begin{cases}1, & i=j=1 \\
0, & \text { otherwise; }\end{cases} \right. \\
x_{i j}=\left\{\begin{array}{ll}
3, & i=j=1 \\
6, & i=2, j=1 \\
0, & \text { otherwise; }
\end{array} \quad y_{i j}= \begin{cases}\frac{1}{15}, & i=j=1 \\
\frac{2}{15}, & i=1, j=2 \\
0, & \text { otherwise. }\end{cases} \right.
\end{array}
$$

Then $E$ and $F$ are algebraically equivalent projections (via $X$ and $Y$ ). However, it is easy to see that $E$ and $F$ are not *-equivalent (since there is no rational number whose square is $1 / 5)$.

Lemma 1.1. [10, Lemma 2, p. 74]. Let $\mathscr{R}$ be a $*$-regular ring with unit which satisfies the condition

$$
x_{1}^{*} x_{1}+\cdots+x_{n}^{*} x_{n}=0 \text { implies } x_{1}=\cdots=x_{n}=0
$$

$(n=1,2, \ldots), x_{i} \in \mathscr{R}(i=1,2, \ldots, n)$. Then the centre of $\mathscr{R}$ contains a subfield isomorphic to the field of rational numbers.

In particular, if $\mathscr{R}$ is an irreducible $*$-regular Baer ring which has order $k$ for some $k \geq 4$ and possesses property $(P U)$, then the following conditions are satisfied (this was proved by von Neumann in [6]; for a proof, see [9, Theorem 4, p. 220]):

(a) If $x, y \in \mathscr{R}$ are both positive, then $x+y$ is positive.

(b) If $x, y \in \mathscr{R}$ are both positive and $x+y=0$, then $x=y=0$.

It is easily seen that these two conditions imply the condition in the statement of Lemma 1.1.

We shall be concerned mainly with irreducible $*$-regular Baer rings with order $k, k \geq 4$. These arise naturally as the coordinatizing rings of those continuous geometries which admit an orthocomplementation. Conversely, the projection lattice of an irreducible *-regular Baer ring is a continuous geometry with this property. 
Our attention will be focused on the property $(P U)$. This property in an irreducible *-regular Baer ring with order $k$ for some $k \geq 4$ has several equivalent formulations.

THEOREM 1.3. Let $\mathscr{R}$ be an irreducible $*$-regular Baer ring with order $k$ for some $k \geq 4$. The following are equivalent.

(i) For any $e, f \in P(\mathscr{R}), \stackrel{a}{\sim} f \Rightarrow e \stackrel{*}{\sim} f$.

(ii) For any $e, f \in P(\mathscr{R}), e \stackrel{a}{\sim} f \Rightarrow e \stackrel{u}{\sim} f$.

(iii) For any $a \in \mathscr{R}, p_{a} \stackrel{*}{\sim}_{a} p$.

(iv) For any $e, f \in P(\mathscr{R}), e \cup f-f \stackrel{*}{\sim} e-e \cap f$.

(v) If $e \in P(\mathscr{R})$ and $x^{*} x \in e \mathscr{R} e$, then there exists $z \in e \mathscr{R} e$ with $x^{*} x=z^{*} z$.

Proof. Let $R$ denote the normalized rank function of $\mathscr{R}$.

(i) $\Rightarrow$ (ii) we have $e \stackrel{a}{\sim} f$. Hence $1-e \stackrel{a}{\sim} 1-f$ [3, Exercise 2, p. 88]. So for some $w \in \mathscr{R} f, v \in(1-e) \mathscr{R}(1-f)$, we have $w w^{*}=e, w^{*} w=f, v v^{*}=1-e, v^{*} v=1-f$. Put $u=w^{*}+v^{*}$. Then

And

$$
\begin{aligned}
u u^{*} & =\left(w^{*}+v^{*}\right)(w+v) \\
& =w^{*} w+w^{*} v+v^{*} w+v^{*} v \\
& =f+w^{*} e(1-e) v+v^{*}(1-e) e w+1-f \\
& =1 \\
& =u^{*} u
\end{aligned}
$$

$$
\begin{aligned}
u e u^{*} & =\left(w^{*}+v^{*}\right) e(w+v) \\
& =w^{*} e w+w^{*} e v+v^{*} e w+v^{*} e v \\
& =f+w^{*} e(1-e) v+v^{*}(1-e) e w+v^{*}(1-e) e v \\
& =f .
\end{aligned}
$$

(ii) $\Rightarrow$ (iii) For any $a \in \mathscr{R}, p_{a} \stackrel{a}{\sim}_{a} p[5$, Exercise 7, p. 38]. Hence there exists unitary $u \in \mathscr{R}$ with $u p_{a} u^{*}={ }_{a} p$. Put $w=u p_{a}$. Then $w w^{*}={ }_{a} p, w^{*} w=p_{a}$.

(iii) $\Rightarrow$ (iv) Let $e, f \in P(\mathscr{R})$ and let $g=e \cup f-f, h=e-e \cap f$. Then $g \stackrel{a}{\sim} h$ [4, Lemma 1 , p. 525]. It follows that $1-g \stackrel{a}{\sim} 1-h$ and that there exists $x \in g \mathscr{R} h$, $y \in h \mathscr{R} g, u \in(1-g) \mathscr{R}(1-h), v \in(1-h) \mathscr{R}(1-g)$ with $x y=g, y x=h, u v=1-g$, $v u=1-h$.

Let $s=x+u, t=y+v$. Then

$$
\begin{aligned}
s t & =(x+u)(y+v) \\
& =x y+x v+u y+u v \\
& =g+x h(1-h) v+u(1-h) h y+1-g \\
& =1 \\
& =t s .
\end{aligned}
$$


Hence $t=s^{-1}$ and

$$
\begin{aligned}
s^{-1} g s & =(y+v) g(x+u) \\
& =y g x+y g u+v g x+v g u \\
& =h+y g(1-g) u+v(1-g) g x+v(1-g) g u \\
& =h .
\end{aligned}
$$

We therefore have $g \cdot g s=g s, g s \cdot s^{-1}=g$. So $p_{g s}=g$. Also, $g s \cdot h=g s, s^{-1} \cdot g s=h$. So ${ }_{g s} p=h$. It follows that $g \stackrel{*}{\sim} h$.

(iv) $\Rightarrow$ (i) Suppose that $e, f \in P(\mathscr{R})$ with $\stackrel{a}{\sim} f, e \perp f$. There exists $g \in P(\mathscr{R})$ with $e+f=e \cup g=f \cup g$ and $e \cap g=f \cap g=0$ [7, Theorem 15.3(c), p. 215]. We will show that $e \stackrel{*}{\sim} g$. Since $e \perp f$, we may write $e^{\perp}=f+f^{\prime}$ where $f^{\prime}$ is the orthogonal complement of $f$ in $e^{\perp}$.

$$
\begin{aligned}
g \cap e^{\perp} & =g \cap\left(f+f^{\prime}\right) \\
& =g \cap\left(f \cup\left(f^{\prime} \cap(g \cup f)\right)\right) \\
& =g \cap\left(f \cup\left(f^{\prime} \cap(e+f)\right)\right) \\
& =g \cap(f \cup 0) \\
& =g \cap f \\
& =0 .
\end{aligned}
$$

So $g-g \cap e^{\perp}=g$. Also,

$$
\begin{aligned}
g \cup e^{\perp} & =g \cup\left(f+f^{\prime}\right) \\
& =g \cup\left(f \cup f^{\prime}\right) \\
& =(g \cup f) \cup f^{\prime} \\
& =(e \cup f) \cup f^{\prime} \\
& =e \cup\left(f \cup f^{\prime}\right) \\
& =e \cup e^{\perp}=1 .
\end{aligned}
$$

So $g \cup e^{\perp}-e^{\perp}=e$. Hence $e \stackrel{*}{\sim} g$. Similarly, $g \stackrel{*}{\sim} f$; so $e^{\prime} \sim$ f. Suppose now that $e \stackrel{a}{\sim} f$ and $R(e)=R(f) \leq 1 / 4$. There exists $h \leq 1-e \cup f$ with $e \stackrel{a}{\sim} h \stackrel{a}{\sim} f$. From the above (since $h \perp e, f), e \stackrel{*}{\sim} h \stackrel{*}{\sim} f$; so $e^{\stackrel{*}{\sim}} f$. Suppose finally that $e \stackrel{a}{\sim} f$ without restriction. We may write

$$
\begin{aligned}
& e=e_{1}+\cdots+e_{k}, \\
& f=f_{1}+\cdots+f_{k},
\end{aligned}
$$

with $e_{i} \perp e_{j}, f_{i} \perp f_{j}(i \neq j)$, and $R\left(e_{i}\right)=R\left(f_{i}\right) \leq 1 / 4(i=1,2, \ldots, k)$. It follows that $e_{i} \stackrel{*}{\sim} f_{i}$ and that $\stackrel{*}{\sim} f$ since $*$-equivalence is finitely additive [5, Theorem 25, p. 33]. (i) $\Leftrightarrow(v)$. This follows from [9, Theorem 1, p. 215].

This completes the proof. 
Property $(P U)$ may be regarded as that property which an irreducible $*$-regular Baer ring with order $k, k \geq 4$, must have in order for the notions of equality in rank, perspectivity, algebraic equivalence, $*$-equivalence, and unitary equivalence of projections to coincide.

THEOREM 1.4. Let $\mathscr{R}$ be an irreducible *-regular Baer ring with order $k, k \geq 4$. Let $R$ denote the normalized rank of $\mathscr{R}$. Then $\mathscr{R}$ has property $(P U)$ if and only if the following are equivalent for $e, f \in P(\mathscr{R})$.

(i) $R(e)=R(f)$.

(ii) $e \sim f$.

(iii) $e \stackrel{a}{\sim} f$.

(iv) $e \stackrel{*}{\sim} f$.

(v) $e \stackrel{u}{\sim} f$.

Proof. If these condition are equivalent, in particular, (iii) $\Rightarrow$ (iv); that is, $\mathscr{R}$ has property $(P U)$.

Conversely, suppose that $\mathscr{R}$ has property $(P U)$. Then (iii) $\Rightarrow$ (iv).

(iv) $\Rightarrow$ (v) We have $e \stackrel{*}{\sim} f$ and (using property $(P U)$ ) $1-e \stackrel{*}{\sim} 1-f$. It follows as in the proof of (i) $\Rightarrow$ (ii) of Theorem 1.3 and from property $(P U)$ that $e \stackrel{u}{\sim} f$.

(v) $\Rightarrow$ (i) This follows from [7, Theorem 17.1(d), p. 224].

(i) $\Rightarrow$ (ii) This follows from [7, Theorem 6.9, p. 52].

(ii) $\Rightarrow$ (iii) This follows from [7, Theorem 15.3(a), p. 215].

This completes the proof.

2. The matrix ring over a regular ring. We denote by $\mathscr{R}_{n}$ the ring of $n \times n$ matrices over a ring $\mathscr{R}$. Von Neumann showed that $\mathscr{R}_{n}$ is regular if and only if $\mathscr{R}$ is regular. If $\mathscr{R}$ is a regular ring with unit and with normalized rank function $R$, then $\mathscr{R}_{n}$ admits a unique normalized rank function $R_{n}^{\prime}$ [2]. If $\mathscr{R}$ is complete with respect to the metric of $R, \mathscr{R}_{n}$ is complete with respect to the metric of $R_{n}^{\prime}$. We will find it convenient to work with the rank function $R_{n}=n R_{n}^{\prime}$ which has the property:

$$
R_{n}(E(e))=n R(e)
$$

whenever $e$ is idempotent in $\mathscr{R}$ and $E(e)$ is the matrix in $\mathscr{R}_{n}$ which has $e$ for all diagonal entries and zeros elsewhere. We denote by $E_{i}(a)$ the matrix in $\mathscr{R}_{n}$ which has $a$ for $i$ th diagonal entry and zeros elsewhere and by $E(a)$ the matrix $\sum_{i=1}^{n} E_{i}(a)$. We note that if $e$ is idempotent in $\mathscr{R}$, then

$$
R_{n}\left(E_{i}(e)\right)=\frac{1}{n} R_{n}(E(e))=R(e) .
$$

It is easy to see that $\mathscr{R}_{n}$ is irreducible if $\mathscr{R}$ is irreducible.

If $\mathscr{R}$ is $*$-regular, $\mathscr{R}_{n}$ may fail to be $*$-regular. In fact, $\mathscr{R}_{n}$ is $*$-regular if and only if $\mathscr{R}$ has the property

$$
x_{1}^{*} x_{1}+\cdots+x_{n}^{*} x_{n}=0 \text { implies } x_{1}=\cdots=x_{n}=0
$$


$x_{i} \in \mathscr{R}(i=1,2, \ldots, n)$ (see [5, Exercise 8, p. 38]). In particular, if $\mathscr{R}$ is an irreducible *-regular Baer ring which has order $k$ for some $k \geq 4$ and possesses property $(P U), \mathscr{R}_{n}$ is $*$-regular.

3. Lifting property $(P U)$ from $\mathscr{R}$ to $\mathscr{R}_{n}$. Throughout this section, $\mathscr{R}$ is an irreducible $*$-regular Baer ring. We suppose that $\mathscr{R}$ has order $k$ for some $k \geq 4$. The object of this section is to raise property $(P U)$ from $\mathscr{R}$ to $\mathscr{R}_{2}$ and thence to $\mathscr{R}_{n}(n=3,4, \ldots) . \mathscr{R}$ admits a normalized rank function and our analysis is carried out in terms of the rank function. The completeness of $\mathscr{R}$ is assumed only to ensure that comparability of projections is at hand: for $e, f \in P(\mathscr{R})$, either there exists $e_{1} \in P(\mathscr{R})$ with $e_{1} \leq e$ and $e_{1} \sim f$ or there exists $f_{1} \in P(\mathscr{R})$ with $f_{1} \leq f$ and $e \sim f_{1}$. Completeness is not a necessary condition for comparability, and comparability could be assumed outright. In this case, $\mathscr{R}$ may fail to admit a rank function; nevertheless, the analysis can be carried out by comparing projections with a fixed reference projection.

LeMma 3.1. Let $E=\left(a_{i j}\right) \in P\left(\mathscr{R}_{2}\right)$ and suppose that $0<R_{2}(E) \leq 1 / 4$. Then there exists unitary $U \in \mathscr{R}_{2}$ and $e \in P(\mathscr{R})$ with $U E U^{*}=E_{1}(e)$.

Proof. From $\left[2, \S 7.2\right.$, p. 332] it follows that $R\left(a_{i j}\right) \leq 1 / 4(i, j=1,2)$. Let

$$
p_{a_{11}} \cup p_{a_{12}}=f, \quad p_{a_{21}} \cup p_{a_{22}}=g .
$$

If $f=0$, then $a_{11}=a_{12}=a_{21}^{*}=a_{21}=0$ and $a_{22}=g$; if $g=0$, then $a_{22}=a_{21}=a_{12}^{*}=a_{12}=0$ and $a_{11}=f$; in either case, the problem is trivial. Assume, then, that $f, g \neq 0$. We have $a_{11}=f a_{11}, a_{12}=f a_{12}, a_{21}=g a_{21}, a_{22}=g a_{22}$. Also, $R(f), R(g) \leq 1 / 2$, so there exists unitary $u \in \mathscr{R}$ with $u f u^{*}=f_{1} \leq(1-g)$. It follows that $g u f=0$. Define

$$
U=\left[\begin{array}{cc}
(1-g) u & g \\
g u & 1-g
\end{array}\right] \text {. }
$$

Easy calculations show that $U$ is unitary in $\mathscr{R}_{2}$ and that

for some $x, y \in \mathscr{R}$. Thus

$$
U E=\left[\begin{array}{ll}
x & y \\
0 & 0
\end{array}\right]
$$

$$
\begin{aligned}
U E U^{*} & =(U E)(U E)^{*} \\
& =\left[\begin{array}{ll}
x & y \\
0 & 0
\end{array}\right]\left[\begin{array}{ll}
x^{*} & 0 \\
y^{*} & 0
\end{array}\right] \\
& =\left[\begin{array}{cc}
x x^{*}+y y^{*} & 0 \\
0 & 0
\end{array}\right] .
\end{aligned}
$$

It is obvious that $e=x x^{*}+y y^{*} \in P(\mathscr{R})$. This completes the proof of the lemma.

LEMma 3.2. Let $E, F \in P\left(\mathscr{R}_{2}\right)$ and suppose that $R_{2}(E)=R_{2}(F) \leq 1 / 4$. Then there exists unitary $U \in \mathscr{R}_{2}$ with $U E U^{*}=F$. 
Proof. There exists unitaries $U_{1}, U_{2} \in \mathscr{R}$ and $e, f \in P(\mathscr{R})$ with $U_{1} E U_{1}^{*}=E_{1}(e)$, $U_{2} F U_{2}^{*}=E_{1}(f)$. Now $R(e)=R_{2}\left(E_{1}(e)\right)=R_{2}(E)=R_{2}(F)=R_{2}\left(E_{1}(f)\right)=R(f)$, so there exists unitary $u \in \mathscr{R}$ with $u e u^{*}=f$. Define $U=U_{2}^{*} \cdot E(u) \cdot U_{1}$. Then $U$ is unitary in $\mathscr{R}_{2}$ and $U E U^{*}=F$. This completes the proof of the lemma.

LeMma 3.3. Let $E, F \in P\left(\mathscr{R}_{2}\right)$ with $R_{2}(E)=R_{2}(F)$. Then there exists $W \in E \mathscr{R}_{2} F$ with $E=W W^{*}, F=W^{*} W$.

Proof. We may write

$$
\begin{aligned}
& E=E_{1}+\cdots+E_{k}, \\
& F=F_{1}+\cdots+F_{k},
\end{aligned}
$$

with $E_{i} \perp E_{j}, F_{i} \perp F_{j}(i \neq j)$, and $R_{2}\left(E_{i}\right)=R_{2}\left(F_{i}\right) \leq 1 / 4(i=1,2, \ldots, k)$. By Lemma 3.2 , there exists unitary $U_{i} \in \mathscr{R}_{2}$ with $U_{i} E_{i} U_{i}^{*}=F_{i}(i=1,2, \ldots, k)$. So $E_{i} \stackrel{*}{\sim} F_{i}$ (via $W_{i}=E_{i} U_{i}^{*}$ ). Now $*$-equivalence is finitely additive [5, Theorem 25, p. 33]. This completes the proof of the lemma.

LEMMA 3.4. Let $m=2^{k}$ for some $k \geq 1$, and let $E, F \in P\left(\mathscr{R}_{m}\right)$ with $R_{m}(E)=R_{m}(F)$. Then there exists $W \in E \mathscr{R}_{m} F$ with $E=W W^{*}, F=W^{*} W$.

Proof. We observe that $\mathscr{R}_{2^{k}} \stackrel{*}{\cong}\left(\mathscr{R}_{2^{k-1}}\right)_{2}$. The lemma now follows by a simple induction from Lemma 3.3.

Corollary. Property $(P U)$ lifts from $\mathscr{R}$ to $\mathscr{R}_{m}, m=2^{k}$.

Proof. Let $E, F \in P\left(\mathscr{R}_{m}\right)$ with $E \stackrel{a}{\sim} F$. Then $E=X Y ; F=Y X$ for some $X \in E \mathscr{R}_{m} F$, $Y \in F \mathscr{R}_{m} E$. So

$$
\begin{aligned}
R_{m}(E) & =R_{m}(X Y) \leq R_{m}(Y)=R_{m}(F Y) \leq R_{m}(F) \\
& =R_{m}(Y X) \leq R_{m}(X)=R_{m}(E X) \leq R_{m}(E) .
\end{aligned}
$$

Hence $R_{m}(E)=R_{m}(F)$ and the corollary follows from the lemma.

It follows readily from condition (v) in the statement of Theorem 1.3 that if $e \in P(\mathscr{R})$, then $e \mathscr{R} e$ has property $(P U)$. We prove the following lemma.

LEMMA 3.5. Let $\mathscr{R}$ be a $*$-regular ring with normalized rank function $R$ and the property: projections with equal rank are *-equivalent. Let e, $f \in P(\mathscr{R})$ with $R(e)=$ $R(f)$ and let $e \cup f=g$. Then there exists unitary $u \in \mathscr{R}$ with ueu* $=f$ and $u h=h$ for all $h \leq(1-g)$.

Proof. Let $e_{1}=g-e, f_{1}=g-f$. Then

$$
\begin{aligned}
R\left(e_{1}\right) & =R(g-e)=R(g)-R(e) \\
& =R(f)-R(e)=R(f-e)=R\left(f_{1}\right) .
\end{aligned}
$$

There exist unitaries $v, v_{1} \in \mathscr{R}$ with $v_{1} e_{1} v_{1}^{*}=f_{1}, v e v^{*}=f$. Define $w=v e+v_{1} e_{1}$. Then

$$
\begin{aligned}
w w^{*} & =\left(v e+v_{1} e_{1}\right)\left(e v^{*}+e_{1} v_{1}^{*}\right) \\
& =v e v^{*}+v e e_{1} v_{1}^{*}+v_{1} e_{1} e v^{*}+v_{1} e_{1} v_{1}^{*} \\
& =f+f_{1}=g .
\end{aligned}
$$


Similarly, $w^{*} w=g$ and $w^{*} w^{*}=f$. Let $u=w+1-g$. Then clearly $u$ is unitary and $u e u^{*}=w_{e w}^{*}=f$.

Moreover, for $h \leq(1-g)$,

This completes the proof.

$$
\begin{aligned}
u h & =u(1-g) h \\
& =(w+1-g)(1-g) h \\
& =(w(1-g)+1-g) h \\
& =(w g(1-g)+1-g) h \\
& =(1-g) h=h .
\end{aligned}
$$

THEOREM 3.1. Let $\mathscr{R}$ be an irreducible *-regular Baer ring with order $k, k \geq 4$, which satisfies property $(P U)$. Then property $(P U)$ lifts from $\mathscr{R}$ to $\mathscr{R}_{n}(n=1,2, \ldots)$.

Proof. Choose $k$ so that $2^{k}=m \geq n$. Let $P=\left(p_{i j}\right) \in P\left(\mathscr{R}_{m}\right)$ be defined by

$$
p_{i j}= \begin{cases}1, & i=j \leq n \\ 0, & \text { otherwise. }\end{cases}
$$

Then $P \mathscr{R}_{m} P \stackrel{*}{\cong} \mathscr{R}_{n}$. Now $\mathscr{R}_{m}$ has property $(P U)$ by Lemma 3.4 . Hence $\mathscr{R}_{n}$ has property $(P U)$ by the remark preceding Lemma 3.5. This completes the proof of the theorem.

4. The inductive limit of a system of rings. Let $(I, \leq)$ be a partially ordered set which is directed up (i.e., for $i, j \in I$ there is a $k \in I$ with $i \leq k, j \leq k$ ). Suppose that for each $i \in I, \mathscr{R}_{i}$ is a ring and that for each $i, j$ with $i \leq j$ there is a ring homomorphism $\Psi_{j i}: \mathscr{R}_{i} \rightarrow \mathscr{R}_{j}$ such that whenever $i \leq j \leq k$, we have

$$
\Psi_{k j} \Psi_{j i}=\Psi_{k i} \text {. }
$$

Let $S$ be the subset of $\left(\bigcup_{i \in I} \mathscr{R}_{i}\right) \times I$ described by $\left\{(a, i): i \in I\right.$ and $\left.a \in \mathscr{R}_{i}\right\}$. We define a relation $\rho \subset S \times S$ by $(a, i) \rho(b, j)$ if there is a $(c, k) \in S$ with $i \leq k, j \leq k$ and $\Psi_{k i} a=\Psi_{k j} b=c$. The relation $\rho$ is clearly an equivalence relation on $S$. The equivalence classes of $S$ form a ring, which we denote by $\mathscr{R}$ and call the inductive limit of the system $\left(I, \leq, \mathscr{R}_{i}, \Psi_{j i}\right)$ with respect to the following operations. If $\rho_{(a, i)}$ denotes the equivalence class of $(a, i) \in S$, addition and multiplication in $\mathscr{R}$ are defined by the rules:

(1) $\rho_{(a, i)}+\rho_{(b, j)}=\rho_{\left(\Psi_{k i} a+\Psi_{k j} b, k\right)}$

(2) $\left.\rho_{(a, i)} \cdot \rho_{(b, j)}=\rho_{\left(\Psi_{k i} a\right.} \cdot \Psi_{k j} b, k\right)$,

where $i \leq k, j \leq k$.

We note the following (see $[2, \S 9])$.

(i) If each $\mathscr{R}_{i}$ is regular, $\mathscr{R}$ is regular.

(ii) If each $\Psi_{j i}$ is injective, then the mapping $a \mapsto \rho_{(a, i)}$ is an injective ring embedding of $\mathscr{R}_{i}$ in $\mathscr{R}$. 
(iii) If each $\mathscr{R}_{i}$ is a regular ring with rank function $R_{i}: \mathscr{R}_{i} \rightarrow[0,1] \dagger$ and each $\Psi_{j i}$ preserves the rank, then the function $R: \mathscr{R} \rightarrow[0,1]$ given by

$$
R\left(\rho_{(a, i)}\right)=R_{i}(a)
$$

is a rank function on $\mathscr{R}$.

(iv) If each $\mathscr{R}_{i}$ is irreducible and the $\Psi_{j i}$ 's are injective, $\mathscr{R}$ is irreducible.

(v) If each $\mathscr{R}_{i}$ is $*$-regular (we denote the involution in each $\mathscr{R}_{i}$ by $*$ ) and each $\Psi_{j i}$ preserves the involution, then the mapping $*: \mathscr{R} \rightarrow \mathscr{R}$ defined by

$$
\rho_{(a, i)}^{*}=\rho_{\left(a^{*}, i\right)}
$$

is an involution with respect to which $\mathscr{R}$ is $*$-regular.

(vi) If $I$ has infinitely many elements and each $\mathscr{R}_{i}$ is a rank ring, $\mathscr{R}$ need not be complete in rank metric even if each $\mathscr{R}_{i}$ is complete.

It follows that if each $\mathscr{R}_{i}$ is an irreducible $*$-regular Baer ring, $\mathscr{R}$ is an irreducible $*$-regular rank ring with unit. We will see in the next section that in this case the completion $\mathscr{R}^{\wedge}$ of $\mathscr{R}$ in rank metric is again an irreducible $*$-regular Baer ring.

5. The completion of a regular rank ring. Let $\mathscr{R}$ be a regular ring with unit and rank function $R$. We denote by $N$ the set of positive integers and by $X_{n \in N} \mathscr{R}$ the collection of mappings

$$
\begin{aligned}
\alpha: N & \rightarrow \mathscr{R} \\
n & \mapsto \alpha_{n} .
\end{aligned}
$$

Let $T$ be the subset of $X_{n \in N} \mathscr{R}$ whose elements $\alpha$ satisfy $R\left(\alpha_{n}-\alpha_{m}\right) \rightarrow 0$ as $n, m \rightarrow \infty$. We define a relation $\equiv \subset T \times T$ by $\alpha \equiv \beta$ if $R\left(\alpha_{n}-\beta_{n}\right) \rightarrow 0$ as $n \rightarrow \infty$. The relation $\equiv$ is clearly an equivalence relation on $T$. The equivalence classes of $\equiv$ form a ring, which we denote by $\mathscr{R}^{\wedge}$ and call the completion of $\mathscr{R}$, with respect to the usual pointwise operations.

We extend the rank function on $\mathscr{R}$ to a function (again denoted by $R$ ) on $\mathscr{R}^{\wedge}$ in the following way. Let $a \in \mathscr{R}^{\wedge}$ and suppose that $\alpha \in a$; then

$$
R(a)=\lim _{n \rightarrow \infty} R\left(\alpha_{n}\right)
$$

Let $a \in \mathscr{R}$; we denote the equivalence class of the mapping $\alpha: N \rightarrow \mathscr{R}$ given by $\alpha_{n}=a(n=1,2, \ldots)$ by $\hat{a}$, and we define $\wedge: \mathscr{R} \rightarrow \mathscr{R}^{\wedge}$ by $a \mapsto \hat{a}$.

THEOREM 5.1. [1, 3.6 and 3.7, p. 716].

(i) $\mathscr{R}^{\wedge}$ is a regular ring.

(ii) $R$ is a rank function on $\mathscr{R}^{\wedge}$.

(iii) $\mathscr{R}^{\wedge}$ is complete with respect to its rank metric. p. 11.

$\dagger$ In the case of matrix rings this notation conflicts with the notation $R_{i}=i R_{i}^{\prime}$ introduced on 
(iv) The mapping $\wedge: \mathscr{R} \rightarrow \mathscr{R}^{\wedge}$ is a ring isomorphic embedding of $\mathscr{R}$ into $\mathscr{R}^{\wedge}$ preserving rank.

(v) $\wedge(\mathscr{R})=\mathscr{R}^{\wedge}$ if and only if $\mathscr{R}$ is complete in rank metric.

We note the following.

(i) If $\mathscr{R}$ is a $*$-regular ring, we extend the involution (again denoted by $*$ ) to $\mathscr{R}^{\wedge}$ as follows. Let $a \in \mathscr{R}^{\wedge}$ and let $\alpha \in a$. Define $\alpha^{\prime}: N \rightarrow \mathscr{R}$ by

$$
\alpha_{n}^{\prime}=\alpha_{n}^{*} \quad(n=1,2, \ldots) .
$$

Then $\alpha^{\prime} \in T$. If $a^{*}$ denotes the equivalence class of $\alpha^{\prime}$, then the mapping $a \mapsto a^{*}$ is the required extension of the involution to $\mathscr{R}^{\wedge}$. Moreover, $\mathscr{R}^{\wedge}$ is *-regular.

(ii) If $\mathscr{R}$ is irreducible, so is $\mathscr{R}^{\wedge}$ [3, Lemma 3(ii), p. 479].

We have, then, that if $\mathscr{R}$ is an irreducible $*$-regular rank ring with unit, $\mathscr{R}^{\wedge}$ is an irreducible *-regular Baer ring. Combining this with the results of the previous section, we have that if $\left(I, \leq, \mathscr{R}_{i}, \Psi_{j i}\right)$ is a system of irreducible *-regular Baer rings, then the completion $\mathscr{R}^{\wedge}$ of

$$
\mathscr{R}=\lim _{\rightarrow}\left(I, \leq, \mathscr{R}_{i}, \Psi_{j i}\right)
$$

is an irreducible $*$-regular Baer ring. If in addition each $\mathscr{R}_{i}$ has property $(P U)$, it follows readily that $\mathscr{R}$ has property $(P U)$. In the next section we prove, under the additional assumption of comparability in $\mathscr{R}$, that property $(P U)$ persists from $\mathscr{R}$ to $\mathscr{R}^{\wedge}$.

6. Extension of property $(P U)$ from incomplete $\mathscr{R}$ to complete $\mathscr{R}^{\wedge}$. Throughout this section, $\mathscr{R}$ is an irreducible $*$-regular ring with unit and normalized rank function $R$. We suppose that $\mathscr{R}$ has order $k$ for some $k \geq 4$ and possesses property $(P U)$. Since we are assuming $\mathscr{R}$ to be not complete, Theorem 1.4 does not apply. However,

$$
e \stackrel{a}{\sim} f \Rightarrow R(e)=R(f)
$$

does (as always in a rank ring) hold. If the rank function is to be useful as a means of analysing $\mathscr{R}$ some kind of converse of this is needed. The rank function does not itself give an idea of relative size; if $e, f \in P(\mathscr{R})$ and $R(e)<R(f), e$ cannot usefully be thought of as being smaller than $f$ unless there is an image of $e$ (having the same size as $e$ in terms of the appropriate notion of size) inside $f$. To ensure that $\mathscr{R}$ is tractible and that no pathology arises we will assume in all that follows that comparability holds in $\mathscr{R}$ :

If $e, f \in P(\mathscr{R})$ and $R(e) \leq R(f)$, then there exists $f_{1} \in P(\mathscr{R})$ with $f_{1} \leq f$ and $e \sim f_{1}$.

A formally weaker approach is to assume in place of property $(P U)$ the condition:

(1) $R(e)=R(f) \Rightarrow e \stackrel{*}{\sim} f$. 
Then the condition:

(2) If $e, f \in P(\mathscr{R})$ and $R(e) \leq R(f)$, then there exists $f_{1} \in P(\mathscr{R})$ with $f_{1} \leq f$ and $R(e)=R\left(f_{1}\right)$

ensures that $\mathscr{R}$ is amenable to analysis. We observe that condition (1) is equivalent here (and in any $*$-regular rank ring) to

$$
R(e)=R(f) \Rightarrow e \stackrel{u}{\sim} f
$$

LEMMA 6.1. $\mathscr{R}$ has comparability if and only if $\mathscr{R}$ satisfies conditions (1) and (2) above.

Proof. Suppose that $\mathscr{R}$ has comparability. Let $e, f \in P(\mathscr{R})$ with $R(e)=R(f)$. There exists $f_{1} \leq f$ with $e \sim f_{1}$. It follows that $e \stackrel{a}{\sim} f_{1}$ [7, Theorem 15.3(a), p. 215] and that $R(e)=R\left(f_{1}\right)$. Since $R\left(f-f_{1}\right)=R(f)-R\left(f_{1}\right)=R(e)-R(e)=0$ we have $f=f_{1}$. That is, $e \stackrel{a}{\sim} f$. Since $\mathscr{R}$ has property $(P U), e \stackrel{*}{\sim} f$. This establishes that $\mathscr{R}$ satisfies condition (1). Now let $e, f \in P(\mathscr{R})$ with $R(e) \leq R(f)$. There exists $f_{1} \leq f$ with $e \sim f_{1}$. Again we have $e \stackrel{a}{\sim} f_{1}$ and $R(e)=R\left(f_{1}\right)$. This establishes that $\mathscr{R}$ satisfies condition (2).

Conversely, suppose that $\mathscr{R}$ satisfies conditions (1) and (2). Let $e, f \in P(\mathscr{R})$ with $R(e) \leq R(f)$. There exists $f_{1} \leq f$ with $R(e)=R\left(f_{1}\right)$. Write

Then

$$
\begin{aligned}
e & =e \cap f_{1}+e^{\prime}, \\
f_{1} & =e \cap f_{1}+f_{1}^{\prime} .
\end{aligned}
$$

$$
\begin{aligned}
R\left(e^{\prime}\right) & =R\left(e-e \cap f_{1}\right)=R(e)-R\left(e \cap f_{1}\right) \\
& =R\left(f_{1}\right)-R\left(e \cap f_{1}\right)=R\left(f_{1}-e \cap f_{1}\right)=R\left(f_{1}^{\prime}\right) .
\end{aligned}
$$

So $e^{\prime} \stackrel{*}{\sim} f_{1}^{\prime}$. Moreover, $e^{\prime} \cap f_{1}^{\prime}=0$. We may apply [7, Theorem 15.3(c), p. 215] to obtain $e^{\prime} \sim f_{1}^{\prime}$. Now [7, Theorem 3.5, p. 20] applies to yield $e \sim f_{1}$. This completes the proof.

Lemma 6.2. Let $e \in P\left(\mathscr{R}^{\wedge}\right)$. Then there exists $\alpha \in a$ with $\alpha_{n} \in P(\mathscr{R})(n=1,2, \ldots)$.

Proof. Let $\beta \in e$. Define $\gamma_{n}=\left(\beta_{n}+\beta_{n}^{*}\right) / 2$ (for the invertibility of 2 , cf. Lemma 1.1). Denote by $\bar{\gamma}_{n}$ the relative inverse of $\gamma_{n}\left[4\right.$, p. 525] and let $e_{n}=P_{\gamma_{n}}$. Then $\gamma_{n} \bar{\gamma}_{n}=e_{n}, e_{n} \gamma_{n}=\gamma_{n}$. We have

$$
\begin{aligned}
R\left(e_{n}-\beta_{n}\right) & =R\left(e_{n}-\gamma_{n}+\gamma_{n}-\beta_{n}\right) \\
& \leq R\left(e_{n}-\gamma_{n}\right)+R\left(\gamma_{n}-\beta_{n}\right) \\
& =R\left(\left(e_{n}-\gamma_{n}\right)^{2}\right)+R\left(\beta_{n}^{*}-\beta_{n}\right)
\end{aligned}
$$

since $e_{n}-\gamma_{n}=\left(e_{n}-\gamma_{n}\right)^{*}$ and $\gamma_{n}-\beta_{n}=\left(\beta_{n}^{*}-\beta_{n}\right) / 2$. Now easy calculations verify

and

$$
\left(e_{n}-\gamma_{n}\right)^{2}=\left(\gamma_{n}-\gamma_{n}^{2}\right)\left(\bar{\gamma}_{n}-1\right)
$$

$$
\gamma_{n}-\gamma_{n}^{2}=\left\{2\left(\beta_{n}-\beta_{n}^{2}\right)+2\left(\beta_{n}-\beta_{n}^{2}\right)^{*}+\left(\beta_{n}-\beta_{n}^{*}\right)\right\} / 4 \text {. }
$$


Hence,

So

$$
\begin{aligned}
R\left(\left(e_{n}-\gamma_{n}\right)^{2}\right) & \leq R\left(\gamma_{n}-\gamma_{n}^{2}\right) \\
& \leq 2 R\left(\beta_{n}-\beta_{n}^{2}\right)+R\left(\beta_{n}-\beta_{n}^{*}\right) .
\end{aligned}
$$

$$
R\left(e_{n}-\beta_{n}\right) \leq 2\left\{R\left(\beta_{n}-\beta_{n}^{2}\right)+R\left(\beta_{n}-\beta_{n}^{*}\right)\right\}
$$

Since $e$ is a projection, both $R\left(\beta_{n}-\beta_{n}^{2}\right)$ and $R\left(\beta_{n}-\beta_{n}^{*}\right)$ approach zero as $n$ becomes large. Let $\alpha: N \rightarrow \mathscr{R}$ be given by

$$
\alpha_{n}=e_{n} \quad(n=1,2, \ldots) .
$$

Then $\alpha \equiv \beta$, so $\alpha \in e$ and $\alpha_{n} \in P(\mathscr{R})$. This completes the proof.

LEMmA 6.3. Suppose that $e \mathscr{R}=p \mathscr{R}$ and $f \mathscr{R}=q \mathscr{R}$, where $e, f \in P(\mathscr{R})$ and $p, q$ are orthogonal idempotents. Then $R(p-q) \leq 2 R(e-f)$.

Proof. We have

Also,

$$
\begin{array}{ll}
e p=p, & p e=e \\
f q=q, & q f=f .
\end{array}
$$

Hence

$$
p q=p f=0=q p=q e .
$$

So

$$
(p+q)(e-f)(p+q)+(p-q)(e-f)(p-q)=2(p-q) .
$$

$$
R(p-q)=R(2(p-q)) \leq 2 R(e-f) .
$$

This completes the proof.

LEMMA 6.4. Let $e, f \in P(\mathscr{R})$. We have

$$
R(e \cap f)+R(e-f) \geq(R(e)+R(f)) / 2 .
$$

Proof. Write

$$
\begin{aligned}
& e=e^{\prime}+e \cap f, \\
& f=f^{\prime}+e \cap f .
\end{aligned}
$$

Then $e-f=e^{\prime}-f^{\prime}$ and $e^{\prime} \cap f^{\prime}=0$. There exist orthogonal idempotents $p, q \in \mathscr{R}$ with

$$
e^{\prime} \mathscr{R}=p \mathscr{R}, \quad f^{\prime} \mathscr{R}=q \mathscr{R}
$$

[1, Lemma 2.1 (2.12), p. 711]. By Lemma 6.3, $R(p-q) \leq 2 R\left(e^{\prime}-f^{\prime}\right)=2 R(e-f)$. Now $(p-q)^{2}=p^{2}-p q-q p+q^{2}=p+q$ and $p-q=p^{2}-q^{2}=(p-q)(p+q)$. Hence

and

$$
R(p)+R(q)=R(p+q)=R\left((p-q)^{2}\right) \leq R(p-q),
$$

$$
R(p-q)=R(p-q)(p+q)) \leq R(p+q)=R(p)+R(q) .
$$


Combining these, we obtain $R(p-q)=R(p)+R(q)$. Since $R\left(e^{\prime}\right)+R\left(f^{\prime}\right)=R(p)+$ $R(q)$, we have $R\left(e^{\prime}\right)+R\left(f^{\prime}\right) \leq 2 R(e-f)$. Also, $R(e \cap f)=R\left(e-e^{\prime}\right)=R\left(f-f^{\prime}\right)=$ $R(e)-R\left(e^{\prime}\right)=R(f)-R\left(f^{\prime}\right)$. So $2 R(e \cap f)+R\left(e^{\prime}\right)+R\left(f^{\prime}\right)=R(e)+R(f)$. Hence $2 R(e \cap f)+2 R(e-f) \geq R(e)+R(f)$.

This completes the proof.

LEMMA 6.5. Let $e_{1}, e_{2}, f_{1}, f_{2} \in P(\mathscr{R})$ with $R\left(e_{1}\right)=R\left(f_{1}\right), R\left(e_{2}\right)=R\left(f_{2}\right)$, and let unitary $u_{1} \in \mathscr{R}$ be such that $u_{1} e_{1} u_{1}^{*}=f_{1}$. Then there exists unitary $u_{2} \in \mathscr{R}$ with $u_{2} e_{2} u_{2}^{*}=f_{2}$ and

$$
R\left(u_{2}-u_{1}\right) \leq 2\left(R\left(e_{2}-e_{1}\right)+R\left(f_{2}-f_{1}\right)\right) .
$$

Proof. Let $R\left(e_{2}-e_{1}\right)=\xi, R\left(f_{2}-f_{1}\right)=\eta$. Also let $f_{3}=u_{1} e_{2} u_{1}^{*}$. Then $f_{3}-f_{1}=$ $u_{1} e_{2} u_{1}^{*}-u_{1} e_{1} u_{1}^{*}=u_{1}\left(e_{2}-e_{1}\right) u_{1}^{*}$, and $R\left(f_{3}-f_{1}\right)=R\left(e_{2}-e_{1}\right)=\xi$. Hence $R\left(f_{3}-f_{2}\right)=$ $R\left(f_{3}-f_{1}+f_{1}-f_{2}\right) \leq R\left(f_{3}-f_{1}\right)+R\left(f_{2}-f_{1}\right)=\xi+\eta$. Write

$$
\begin{aligned}
& f_{2}=f_{2} \cap f_{3}+f_{2}^{\prime}, \\
& f_{3}=f_{2} \cap f_{3}+f_{3}^{\prime} .
\end{aligned}
$$

Then $R\left(f_{3}^{\prime}-f_{2}^{\prime}\right)=R\left(f_{3}-f_{2}\right) \leq \xi+\eta$. Also, $R\left(f_{3}\right)=R\left(e_{2}\right)=R\left(f_{2}\right)$. So $R\left(f_{3}^{\prime}\right)=R\left(f_{2}^{\prime}\right)$. Put $f_{2}^{\prime} \cup f_{3}^{\prime}=g$. Then by Lemma 3.5 , there exists unitary $u_{3} \in \mathscr{R}$ with $u_{3} f_{2}^{\prime} u_{3}^{*}=f_{3}^{\prime}$ and $u_{3} h=h$ for all $h \leq 1-g$. Now $f_{2}^{\prime} \leq 1-f_{2} \cap f_{3}$ and $f_{3}^{\prime} \leq 1-f_{2} \cap f_{3}$, so $f_{2}^{\prime} \cup f_{3}^{\prime}=$ $g \leq 1-f_{2} \cap f_{3}$. Hence $1-g \geq f_{2} \cap f_{3}$ and $u_{3}\left(f_{2} \cap f_{3}\right)=f_{2} \cap f_{3}$. Therefore,

$$
\begin{aligned}
u_{3} f_{2} u_{3}^{*} & =u_{3}\left(f_{2}^{\prime}+f_{2} \cap f_{3}\right) u_{3}^{*} \\
& =u_{3} f_{2}^{\prime} u_{3}^{*}+u_{3}\left(f_{2} \cap f_{3}\right) u_{3}^{*} \\
& =f_{3}^{\prime}+f_{2} \cap f_{3} \\
& =f_{3} .
\end{aligned}
$$

Moreover, $\quad\left(1-u_{3}\right)(1-g)=1-g-(1-g)=0$. So $R\left(1-u_{3}\right)=R\left(\left(1-u_{3}\right) g\right) \leq R(g)$. Now $R(g)=R\left(f_{2}^{\prime} \cup f_{3}^{\prime}\right)=R\left(f_{2}^{\prime}\right)+R\left(f_{3}^{\prime}\right)$, and by Lemma 6.4, $R\left(f_{3}^{\prime}\right)+R\left(f_{2}^{\prime}\right) \leq$ $2 R\left(f_{3}^{\prime}-f_{2}^{\prime}\right)$. Hence $R(g) \leq 2 R\left(f_{3}^{\prime}-f_{2}^{\prime}\right) \leq 2(\xi+\eta)$. So $R\left(1-u_{3}\right) \leq 2(\xi+\eta)$. We have $u_{3} f_{2} u_{3}^{*}=f_{3}=u_{1} e_{2} u_{1}^{*}$, or $\left(u_{3}^{*} u_{1}\right) e_{2}\left(u_{3}^{*} u_{1}\right)^{*}=f_{2}$. Put $u_{3}^{*} u_{1}=u_{2}$. Then $u_{2}$ is unitary, $u_{2} e_{2} u_{2}^{*}=f_{2}$, and

This completes the proof.

$$
\begin{aligned}
R\left(u_{2}-u_{1}\right) & =R\left(u_{3}^{*} u_{1}-u_{1}\right) \\
& =R\left(\left(1-u_{3}\right)^{*} u_{1}\right) \\
& \leq R\left(1-u_{3}\right) \\
& \leq 2(\xi+\eta) \\
& =2\left(R\left(e_{2}-e_{1}\right)+R\left(f_{2}-f_{1}\right)\right) .
\end{aligned}
$$

LEMMA 6.6. Let $e, f \in P\left(\mathscr{R}^{\wedge}\right)$ with $R(e)=R(f)$. Then there exists unitary $u \in \mathscr{R}^{\wedge}$ with $u e u^{*}=f$. 
Proof. Let $\lambda=R(e)=R(f)$. If both $e$ and $f$ are images under the mapping $\wedge: \mathscr{R} \rightarrow \mathscr{R}^{\wedge}$ of projections in $\mathscr{R}$, there is nothing to prove. We will suppose that $e$ is not the image under ${ }_{\wedge}$ of a projection in $\mathscr{R}$. Let $\alpha_{4} \in e, \beta \in f$ with $\alpha_{n}, \beta_{n} \in P(\mathscr{R})$ $(n=1,2, \ldots)$. Since

$$
\lim _{n \rightarrow \infty} R\left(\alpha_{n}\right)=\lambda=R(e),
$$

there is a subsequence $\left(\alpha_{k_{n}}\right)$ of $\left(\alpha_{n}\right)$ such that $R\left(\alpha_{k_{n}}\right)$ either increases monotonously to $\lambda$ or decreases monotonously to $\lambda$. We will suppose that $R\left(\alpha_{k_{n}}\right)$ decreases monotonously to $\lambda$. Put

Then the mapping

$$
\alpha_{k_{n}}=\gamma_{n} \quad(n=1,2, \ldots)
$$

$$
\begin{aligned}
\gamma: N & \rightarrow \mathscr{R} \\
n & \mapsto \gamma_{n}
\end{aligned}
$$

satisfies $\gamma \equiv \alpha \in e$. Now $R\left(e-\hat{\gamma}_{n}\right) \rightarrow 0$ [1, Theorem 3.7(iii), p. 716], so we may assume that

$$
R\left(e-\hat{\gamma}_{n}\right) \leq 2^{-n} \lambda \quad(n=1,2, \ldots) .
$$

(If this is not the case, we may again select a subsequence of $\left(\gamma_{n}\right)$ for which this is true.) We then have

$$
\begin{aligned}
R\left(\gamma_{n+1}-\gamma_{n}\right) & =R\left(\hat{\gamma}_{n+1}-\hat{\gamma}_{n}\right) \\
& =R\left(\hat{\gamma}_{n+1}-e+e-\hat{\gamma}_{n}\right) \\
& \leq R\left(\hat{\gamma}_{n+1}-e\right)+R\left(\hat{\gamma}_{n}-e\right) \\
& <2^{-(n+1)} \lambda+2^{-n} \lambda \\
& =3.2^{-(n+1)} \lambda .
\end{aligned}
$$

Also, $R\left(e-\hat{\gamma}_{n}\right) \geq\left|R(e)-R\left(\gamma_{n}\right)\right|=R\left(\gamma_{n}\right)-R(e)$. So $R\left(\gamma_{n}\right) \leq R(e)+R\left(e-\hat{\gamma}_{n}\right)<\lambda(1+$ $\left.2^{-n}\right)$. We may suppose that $R\left(\beta_{n+1}-\beta_{n}\right)<2^{-(n+1)} \lambda$. Then if $\left(\beta_{k_{n}}\right)$ is any subsequence of $\left(\beta_{n}\right)$, we have $k_{n+1}=k_{n}+l$ for some $l \geq 1$ and since $k_{n} \geq n, 2^{-\left(k_{n}+1\right)} \leq 2^{-(n+1)}$. Thus

$$
\begin{aligned}
R\left(\beta_{k_{n+1}}-\beta_{k_{n}}\right) & =R\left(\beta_{k_{n}+l}-\beta_{k_{n}+l-1}+\cdots+\beta_{k_{n}+1}-\beta_{k_{n}}\right) \\
& \leq R\left(\beta_{k_{n}+l}-\beta_{k_{n}+l-1}\right)+\cdots+R\left(\beta_{k_{n}+1}-\beta_{k_{n}}\right) \\
& <2^{-\left(k_{n}+l\right)} \lambda+\cdots+2^{-\left(k_{n}+1\right)} \lambda \\
& =2^{-\left(k_{n}+1\right)} \lambda\left(1+2^{-1}+\cdots+2^{-(l-1)}\right. \\
& <2^{-(n+1)} \lambda \cdot 2=2^{-n} \lambda .
\end{aligned}
$$

Now since $R\left(\gamma_{n}\right) \downarrow \lambda$ and $\lim _{n \rightarrow \infty} R\left(\beta_{n}\right)=\lambda$, for each $n \in N$ there is a $k_{n} \in N$ with

$$
\frac{1}{2 \lambda-R\left(\gamma_{n}\right) \leq R\left(\beta_{k_{n}}\right) \leq R\left(\gamma_{n}\right) .}
$$


There exists $\beta_{k_{n}}^{\prime} \in P(\mathscr{R})$ with $\beta_{k_{n}}^{\prime} \leq \beta_{k_{n}}$ and $R\left(\beta_{k_{n}}^{\prime}\right)=R\left(\gamma_{n}\right)$. Put

Then the mapping

$$
\beta_{k n}^{\prime}=\delta_{n} \quad(n=1,2, \ldots) \text {. }
$$

$$
\begin{aligned}
\delta: N & \rightarrow \mathscr{R} \\
n & \mapsto \delta_{n}
\end{aligned}
$$

satisfies $\delta \equiv \beta \in f$. Now $-R\left(\beta_{k_{n}}\right) \leq R\left(\gamma_{n}\right)-2 \lambda$. So $R\left(\gamma_{n}\right)-R\left(\beta_{k_{n}}\right) \leq \lambda\left(1+2^{-n}\right)+$ $\lambda\left(1+2^{-n}\right)-2 \lambda=2^{-n+1} \lambda$. Hence

We therefore have

$$
\begin{aligned}
R\left(\beta_{k_{n}}^{\prime}-\beta_{k_{n}}\right) & =R\left(\beta_{k_{n}}^{\prime}\right)-R\left(\beta_{k_{n}}\right) \\
& =R\left(\gamma_{n}\right)-R\left(\beta_{k_{n}}\right) \\
& \leq 2^{-n+1} \lambda .
\end{aligned}
$$

$$
\begin{aligned}
R\left(\delta_{n+1}-\delta_{n}\right) & =R\left(\beta_{k_{n+1}}^{\prime}-\beta_{k_{n}}^{\prime}\right) \\
& \leq R\left(\beta_{k_{n+1}}^{\prime}-\beta_{k_{n+1}}\right)+R\left(\beta_{k_{n+1}}-\beta_{k_{n}}\right)+R\left(\beta_{k_{n}}^{\prime}-\beta_{k_{n}}\right) \\
& \leq 2^{-(n+1)+1} \lambda+2^{-n} \lambda+2^{-n+1} \lambda \\
& =2^{-n} \lambda(1+1+2)=2^{-n+2} \lambda .
\end{aligned}
$$

Summarizing, we have $\gamma \in e, \delta \in f$ with $\gamma_{n}, \delta_{n} \in P(\mathscr{R}), R\left(\gamma_{n}\right)=R\left(\delta_{n}\right)$, and

$$
\left.\begin{array}{l}
\left.R\left(\gamma_{n+1}-\gamma_{n}\right) \leq 3.2^{-(n+1)} \lambda\right) \\
R\left(\delta_{n+1}-\delta_{n}\right) \leq 8.2^{-(n+1)} \lambda
\end{array}\right\} \quad(n=1,2, \ldots)
$$

By induction from Lemma 7.5, there exists a sequence of unitaries $\nu_{n} \in \mathscr{R}$ with $\nu_{n} \gamma_{n} \nu_{n}^{*}=\delta_{n}$ and

$$
\begin{aligned}
R\left(v_{n+1}-v_{n}\right) & \leq 2\left(R\left(\gamma_{n+1}-\gamma_{n}\right)+R\left(\delta_{n+1}-\delta_{n}\right)\right) \\
& \leq 11.2^{-n} \lambda .
\end{aligned}
$$

Therefore, $R\left(v_{m}-v_{n}\right) \rightarrow 0$ as $m, n \rightarrow \infty$ and the mapping

$$
\begin{aligned}
\nu: N & \rightarrow \mathscr{R} \\
n & \mapsto \nu_{n}
\end{aligned}
$$

belongs to $T$. Let $u$ be the equivalence class of $v$. Then, clearly, $u$ is unitary in $\mathscr{R}^{\wedge}$ and $u e u^{*}=f$. This completes the proof.

THEOREM 6.1. Let $\mathscr{R}$ be an irreducible $*$-regular rank ring with order $k, k \geq 4$, in which comparability holds and which satisfies property $(P U)$. Then property $(P U)$ extends from $\mathscr{R}$ to $\mathscr{R}^{\wedge}$.

Proof. Let $e, f \in P(\mathscr{R})$ with $\stackrel{a}{\sim} f$. Then $R(e)=R(f)$ and there exists unitary $u \in \mathscr{R}^{\wedge}$ with $u e u^{*}=f$. Put $w=e u^{*}$. Then $w \in e \mathscr{R}^{\wedge} f$ and

$$
w w^{*}=e, \quad w^{*} w=f .
$$

This completes the proof of the theorem. 
7. An application to inductive limits. Suppose in this section that $\mathscr{R}$ is an irreducible $*$-regular Baer ring which has order $k$ for some $k \geq 4$ and possesses property $(P U)$. We denote as usual by $\mathscr{R}_{n}$ the ring of $n \times n$ matrices over $\mathscr{R}$. We define a relation $/ \subset N \times N$ by: $m / n$ if $n=k m$ for some $k \in N$. Then $(N, /)$ is a partially ordered set which is directed up. If $m / n$, we define an injective ring isomorphism

$$
\Psi_{n m}: \mathscr{R}_{m} \rightarrow \mathscr{R}_{n}
$$

as follows: if $A=\left(a_{i j}\right) \in \mathscr{R}_{m}$, then $\Psi_{n m}(A)$ shall be the matrix $B=\left(b_{p q}\right) \in \mathscr{R}_{n}$ such that

$$
b_{(s-1) k+i,(s-1) k+j}= \begin{cases}a_{i j}, & 1 \leq i, j \leq m, 1 \leq s \leq k \\ 0, & \text { otherwise, }\end{cases}
$$

where $n=k m$ (i.e., $B$ has copies of $A$ down the principal diagonal and zeros elsewhere).

Suppose that $I \subset N$ and that for $m, n \in I$ there exists $k \in I$ such that $m / k, n / k$. We write

$$
\mathscr{R}_{I}=\lim _{\rightarrow}\left(I, /, \mathscr{R}_{n}, \Psi_{n m}\right)
$$

for the inductive limit of the system $\left(I, /, \mathscr{R}_{n}, \Psi_{n m}\right)$. In $\S 5$, we observed that $\mathscr{R}_{I}$ is an irreducible $*$-regular ring with unit and normalized rank function. That $\mathscr{R}_{I}$ has comparability and possesses property $(P U)$ are consequences of the following theorem.

THEOREM 7.1. Let $e, f \in P\left(\mathscr{R}_{I}\right)$. Then:

(1) $R(e)=R(f) \Rightarrow e \stackrel{*}{\sim} f$.

(2) $R(e) \leq R(f)$ implies that there exists $f_{1} \in P\left(\mathscr{R}_{I}\right)$ with $f_{1} \leq f$ and $R(e)=R\left(f_{1}\right)$.

Proof. (1) For some $n, m \in I$ and projections $E \in \mathscr{R}_{n}, F \in \mathscr{R}_{m}$ we have $e=$ $\rho_{(E, n)}, f=\rho_{(F, m)}$. Let $k \in I$ with $n / k, m / k$ and let $\Psi_{k n} E=E^{\prime} \in P\left(\mathscr{R}_{k}\right), \Psi_{k m} F=F^{\prime} \in$ $P\left(\mathscr{R}_{k}\right)$. We have

and

$$
\begin{aligned}
& \rho_{(E, n)}=\rho_{\left(E^{\prime}, k\right)}=e, \\
& \rho_{(F, m)}=\rho_{\left(E^{\prime}, k\right)}=f,
\end{aligned}
$$

$$
\begin{aligned}
& R(e)=R\left(\rho_{\left(E^{\prime} k\right)}\right)=R_{k}\left(E^{\prime}\right), \\
& R(f)=R\left(\rho_{\left(F^{\prime}, k\right)}\right)=R_{k}\left(F^{\prime}\right) .
\end{aligned}
$$

Hence $R_{k}\left(E^{\prime}\right)=R_{k}\left(F^{\prime}\right)$. Since $\mathscr{R}_{k}$ is an irreducible $*$-regular Baer ring, Theorems 1.4 and 3.1 imply that there exists $W \in E^{\prime} \mathscr{R}_{k} F^{\prime}$ with

$$
W W^{*}=E^{\prime}, \quad W^{*} W=F^{\prime} .
$$

Let $\rho_{(W, k)}=w \in \mathscr{R}_{I}$; then $w^{*}=\rho_{\left(W^{*}, k\right)}$ and

$$
\begin{aligned}
& w w^{*}=\rho_{(W, k)} \rho_{\left(W^{*}, k\right)}=\rho_{\left(W W^{*}, k\right)}=\rho_{\left(E^{\prime}, k\right)}=e, \\
& w^{*} w=\rho_{\left(W^{*}, k\right)} \rho_{(W, k)}=\rho_{\left(W W^{*}, k\right)}=\rho_{\left(F^{\prime}, k\right)}=f .
\end{aligned}
$$

This completes the proof of (1). 
(2) Again, we obtain $k \in I$ and projections $E^{\prime}, F^{\prime} \in \mathscr{R}_{k}$ with $\rho_{\left(E^{\prime}, k\right)}=e, \rho_{\left(F^{\prime}, k\right)}=f$. Since $R(e) \leq R(f), R_{k}\left(E^{\prime}\right) \leq R_{k}\left(F^{\prime}\right)$. Since $\mathscr{R}_{k}$ is an irreducible $*$-regular Baer ring, $\mathscr{R}_{k}$ has comparability: there exists $F_{1}^{\prime} \in P\left(\mathscr{R}_{k}\right)$ with $F_{1}^{\prime} \leq F^{\prime}$ and $R_{k}\left(E^{\prime}\right)=R_{k}\left(F_{1}^{\prime}\right)$. Let $\rho_{\left(F^{\prime}, k\right)}=f_{1} \in \mathscr{R}_{I^{\prime}}$. Then $f f_{1}=\rho_{\left(F^{\prime}, k\right)} \rho_{\left(F_{1}^{\prime}, k\right)}=\rho_{\left(F^{\prime} F_{1}^{\prime}, k\right)}=\rho_{\left(F_{1^{\prime}}, k\right)}=f_{1}$, so $f_{1} \leq f$. Moreover, $R(e)=R_{k}\left(E^{\prime}\right)=R_{k}\left(F_{1}^{\prime}\right)=R\left(f_{1}\right)$.

This completes the proof.

We may now employ Theorem 6.1 to obtain that $\mathscr{R}_{I}{ }^{\wedge}$ has property $(P U)$. Under suitable conditions $\mathscr{R}_{I}{ }^{\wedge}$ will be a continuous ring even if $\mathscr{R}$ is discrete.

ACKNOWLEDGement. This work constitutes part of the author's doctoral thesis written at the University of Toronto under the supervision of Professor Israel Halperin. I thank Professor Halperin for his help and encouragement during my time as a Ph.D. student.

\section{REFERENCES}

1. Israel Halperin, Regular rank rings, Can. J. Math. 17 (1965), 709-719.

2. - Extension of the rank function, Studia Math. 27 (1966), 325-335.

3. - von Neumann's manuscript on the inductive limit of regular rings, Can. J. Math. 20 (1968), 477-483.

4. Irving Kaplansky, Any orthocomplemented complete modular lattice is a continuous geometry, Ann. of Math. 61 (1955), 524-541.

5. - Rings of operators (Benjamin, New York, 1968).

6. John von Neumann, Continuous geometries with a transition probability, unpublished manuscript (reviewed by Israel Halperin in the Collected Works of John von Neumann, Pergamon, Elmsford, N.Y., 1962).

7. - Continuous geometry (Princeton University Press, Princeton, 1960).

8. - The non-isomorphism of certain continuous rings, Ann. of Math. 67 (1958), 485-496.

9. N. Prijatelj and I. Vidav, On special *-regular rings, Michigan Math. J. 18 (1971), 213-221.

10. I. Vidav, On some *-regular rings, Acad. Serbe Sci. Publ. Inst. Math. 13 (1959), 73-80.

Department of Mathematics,

UNIVERSITY OF CANTERBURY,

Christchurch 1, New Zealand 